\title{
The cortisol awakening response and major depression: examining the evidence
}

\author{
This article was published in the following Dove Press journal: \\ Neuropsychiatric Disease and Treatment \\ 14 May 2015 \\ Number of times this article has been viewed
}

\author{
Katarina Dedovic ${ }^{1,2}$ \\ Janice Ngiam $^{3}$ \\ 'Department of Psychiatry, Douglas \\ Hospital Research Centre, Montreal, \\ QC, Canada; ${ }^{2}$ Social and Affective \\ Neuroscience Laboratory, University \\ of California, Los Angeles, CA, USA; \\ ${ }^{3}$ Department of Psychology, McGill \\ University, Montreal, QC, Canada
}

\begin{abstract}
A vast body of literature has revealed that dysregulation of the hypothalamicpituitary-adrenal (HPA) stress axis is associated with etiology of major depressive disorder (MDD). There are many ways that the dysregulation of the HPA axis can be assessed: by sampling diurnal basal secretion and/or in response to a stress task, pharmacological challenge, and awakening. Here, we focus on the association between cortisol awakening response (CAR), as one index of HPA axis function, and MDD, given that the nature of this association is particularly unclear. Indeed, in the following selective review, we attempt to reconcile sometimes-divergent evidence of the role of CAR in the pathway to depression. We first examine association of CAR with psychological factors that have been linked with increased vulnerability to develop depression. Then, we summarize the findings regarding the CAR profile in those with current depression, and evaluate evidence for the role of CAR following depression resolution and continued vulnerability. Finally, we showcase longitudinal studies showing the role of CAR in predicting depression onset and recurrence. Overall, the studies reveal an important, but complex, association between CAR and vulnerability to depression.
\end{abstract}

Keywords: HPA axis, MDD, SCN, daily hassles, genetic influence, environmental factors

\section{Introduction}

Dysregulation of the hypothalamic-pituitary-adrenal (HPA) axis both at rest and in times of stress has been associated with major depressive disorder (MDD) in children and in adults. ${ }^{1-5}$ However, the nature, in terms of direction, mechanisms, and predictive capacity, of the association between one specific index of HPA axis function - the cortisol awakening response (CAR) - and MDD still remains unclear. Thus, in the following pages, we review some of the evidence to date linking CAR to vulnerability to depression. The focus on the CAR is warranted, since it has been suggested that CAR is more sensitive to moderate degrees of depression than traditional (albeit, in some cases, currently considered outdated) measures of the HPA axis, such as the dexamethasone (DEX) suppression test (DST). ${ }^{6}$ In addition, CAR is the most straightforward measure of dynamics of the HPA axis that can be obtained reliably in ambulatory setting, ${ }^{7}$ which carries important implications with respect to its potential usage in prevention and intervention efforts. We first provide brief background information on what is CAR, and how certain factors affect it in a healthy population; then, we evaluate CAR's relationship to select factors related to vulnerability to depression and depressive state, as well as prediction of depression in healthy populations and in primary depression populations (current or remitted). Notably, this is not meant to be an exhaustive review but rather a concise overview of the key findings related to CAR and MDD to date. This summary may serve as a platform for developing new studies and furthering our understanding of CAR's role in the depression trajectory.
Correspondence: Katarina Dedovic Dof Psychiatry, Douglas Boulevard Lasalle, Pavilion Perry 4109. Verdun, Montreal, QC, H4H IR3, Canada $\mathrm{Tel}+\mid$ 5I4 76I 6I3I ext 4394 Email katarina.dedovic@mail.mcgill.ca submit your manuscript | www.dovepress.com Dovepress
Neuropsychiatric Disease and Treatment 2015:II II8I-II89 I I8I

(c) (i) (5) 2015 Dedovic and Ngiam. This work is published by Dove Medical Press Limited, and licensed under Creative Commons Attribution - Non Commercial (unported, v3.0) permission from Dove Medical Press Limited, provided the work is properly attributed. Permissions beyond the scope of the License are administered by Dove Medical Press Limited. Information on permission from Dove Medical Press Limited, provided the work is properly attributed. 


\section{Cortisol awakening response}

The CAR is thought to reflect the sensitivity of the HPA axis to a natural challenge (the awakening), but it may also play an important role in other awakening-induced processes such as restoration of consciousness and full alertness, changes in other hormones, and the balance of the immune system, as well as mobilization of the motor system. ${ }^{8-10}$ In healthy participants, CAR involves a sharp increase in cortisol secretion following awakening, which peaks at between 30 minutes and 45 minutes and returns to baseline at 60 minutes postawakening. ${ }^{8,11,12}$ Across healthy adults, the magnitude of peak increase ranges in anywhere from 50\% up to over $100 \%$ compared to baseline. ${ }^{10}$ The CAR is typically assessed on minimum of 2 days, as it has been shown that this increases the reliability of the measure. ${ }^{13,14}$ The change in CAR can be calculated in many ways, including change over time across multiple time points within the first 60 minutes, delta between the peak cortisol level and baseline, or area-under-the-curve (AUC) summary measures which can include AUC with respect to increase from baseline (AUCi or AUCnetto) or from ground or zero (AUCg or AUCtotal).

As CAR is initiated by awakening, its regulatory system is relatively distinct from cortisol regulation throughout the day. Indeed, CAR is influenced by the hypothalamic suprachiasmatic nucleus ( $\mathrm{SCN}$ ), with both the HPA axis and the sympathetic nervous system playing a role in "fine tuning" this direct neural input moving to the adrenal cortex. ${ }^{10,15}$ For example, it has been suggested that interaction between the SCN function and regulatory mechanisms controlling the HPA axis (eg, input from the hippocampus) may underlie the change in adrenal sensitivity to ACTH from being reduced pre-awakening to being increased post-awakening, thus contributing to the cortisol surge in response to awakening. ${ }^{10}$

Studies in healthy population have also shown that certain sociodemographic variables, as well as cortisol sampling procedures and physical health indicators, are related to CAR. For example, women typically show a higher CAR compared to men, ${ }^{11,14}$ although some studies found no difference. ${ }^{16,17}$ Furthermore, older age seems to be associated with lower CAR, ${ }^{17}$ although some authors observed no effect. ${ }^{11,14,18}$ In addition, the day of the week (weekday greater than weekend), ${ }^{19,20}$ time of awakening (earlier awakening time being linked to higher CAR), ${ }^{16,17,21}$ and possibly seasonal influences ${ }^{22}$ have been shown to affect the CAR. Finally, physical health indicators such as general good health have been linked to higher CAR, ${ }^{17}$ while smoking has been associated with both higher, slightly flattened CAR and no difference. ${ }^{12,23,24}$ Therefore, it is important that these factors be controlled for in any study investigating relevance of CAR to vulnerability to depression.

\section{Genetic and environmental influence on CAR}

To date, there have been several studies examining genetic influence on the CAR profile. These studies investigated monozygotic (MZ) and dizygotic (DZ) twin pairs. First, they examined correlations between twins for CAR-related measures of interest in order to assess the similarity between twins, and then, they compared the twin correlations for MZ and DZ twin pairs which provided information about the importance of additive genetic factors, shared environmental factors (environmental influences that make twin siblings similar to each other), and non-shared environmental factors (environmental influences that make twin siblings different from each other). Finally, models were fitted to the data in order to decompose the phenotypic variance of each CAR measure into additive genetic (A), shared environmental (C), and non-shared environmental (E) components; a full univariate ACE model was then compared against AE, CE, and $\mathrm{E}$ models. All studies suggested that the AE model offered the best fit.

In a sample with a wide age range (8-64 years), Wust et al reported heritability estimates of 0.40 for the mean increase (average of 30 minutes, 45 minutes, and 60 minutes compared to baseline) and 0.48 for AUCi; they did not observe any genetic influence on cortisol levels at awakening or daytime cortisol profile. ${ }^{12}$ In a study examining 12 -year-old boys and girls on two school days, significant genetic influences were found for awakening sample $(22 \%, 24 \%)$, second sample taken on average approximately 45 minutes post-awakening $(56 \%, 59 \%)$, and at 12 hours 30 minutes $(30 \%, 21 \%)$ for each day. ${ }^{25}$ Another study on children (9-16 years of age) observed that heritability was highest (60\%) for cortisol levels at approximately 30 minutes post-awakening but less pronounced for measures immediately after awakening (28\%) and low for the evening levels (8\%). ${ }^{26}$ Similarly, genetic model fitting showed heritability at $34 \%$ for cortisol level at awakening and $32 \%$ for cortisol level at 30 minutes after awakening but not for cortisol levels later during the day. ${ }^{27}$

Interestingly, another study of middle-aged men again showed high cortisol heritability estimates for cortisol measures at awakening (0.56), 30 minutes after awakening (0.48), and mean CAR (0.64), followed by cortisol levels at 10:00 hours (0.42) and mean output across the day (0.43); however, these associations emerged only when laboratory visits were considered..$^{28}$ Indeed, twin correlations for the 
samples obtained at home were low. The authors specifically suggested that cortisol levels are correlated in identical twins only when they undergo similar experiences. ${ }^{28}$ For example, given that these adult twins lived and worked apart, their experiences and demands on the HPA axis on the home day would likely differ; on the other hand, the lab environment (that includes elements of novelty and unpredictability) would represent a more standardized and likely heightened demand on the HPA axis. Along those lines, on the test day, only MZ pairs' ratings of stressfulness were significantly correlated, while, on the home day, neither MZ nor DZ twins' ratings of home day's stressfulness were correlated. Overall, these findings revealed that while measures of cortisol related to awakening are under significant genetic influence, genetic factors influence cortisol responses to specific environmental stressors. Given that 1) the CAR is under the regulation of the circadian clock system (which includes the "master" clockthe SCN, and "slave" peripheral clocks operating in all organs and tissues), ${ }^{29}$ 2) the circadian clock system interacts with stress systems, ${ }^{30,31}$ and 3 ) cortisol can influence expression of several circadian clock-related genes and thus plasticity and function of regulatory neural regions, ${ }^{29}$ it is not surprising that contribution of genes to CAR is likely not static.

Overall, these findings highlight possible importance of CAR in capturing both trait and state levels of one's vulnerability to depression.

\section{CAR and psychological traits related to depression vulnerability}

Certain psychological traits such as neuroticism, hopelessness, negative affect, subclinical depression, or family history of MDD have been associated with increased vulnerability to develop depression. ${ }^{32-34}$ In addition, perseverative cognition including rumination and worry has been linked to mood disorders, both depression and anxiety. ${ }^{35}$ Thus, one way to evaluate relevance of CAR with respect to depression is to examine in what way some of these risk factors for depression have been linked with variability in CAR.

\section{Neuroticism}

Results from studies related to association between neuroticism and CAR have been inconsistent. In a study of 15 individuals with high neuroticism scores compared to 15 participants with low scores, Portella et al observed that those with high levels of neuroticism showed increased cortisol levels from +30 minutes to +60 minutes post-awakening. ${ }^{36}$ In addition, in the subjects with high neuroticism scores, the
CAR AUCi for the first 60 minutes was positively associated with neuroticism levels. Similar positive association was also observed between neuroticism levels and CAR defined as percentage difference between maximum and mean morning cortisol in a sample of 69 healthy adults. ${ }^{37}$ On the other hand, another study in healthy Mexican-American adults observed a negative association between cortisol levels at +45 minutes and +60 minutes post-awakening and neuroticism levels, which was moderated by levels of acculturation (to American society). Specifically, among the high acculturated (ie, those who had greater Anglo orientation), greater neuroticism was associated with significantly attenuated CAR profile. ${ }^{38}$ Finally, several studies found no association between CAR measures and neuroticism scores in adolescents, ${ }^{39,40}$ in a large sample with a wide age range ${ }^{41,42}$ and among college samples. ${ }^{43,44}$ Thus, while some authors observed no association between neuroticism and CAR, others found a positive relationship, while others still observed a negative one, which was accentuated by levels of acculturation.

\section{Hopelessness reactivity}

Along the hopelessness theory of depression, ${ }^{45}$ it has been shown that negative attributional style can predict greater depressive symptom reactivity in response to stress. ${ }^{46}$ In addition, hopelessness reactivity can mediate association between neuroticism and depression vulnerability, particularly in those with previous history of depression. ${ }^{47}$ Despite the relative relevance of hopelessness to depression, few studies have investigated its association with CAR. Namely, Sjogren et al assessed hopelessness in 257 men and women, by examining to what degree participants endorsed the following two statements "I feel that it is impossible to reach the goals I would like to strive for" and "The future seems to me to be hopeless, and I cannot believe that things will change for the better"; they found a tendency for negative association with cortisol levels 30 minutes post-awakening, when controlling for relevant sociodemographic factors. ${ }^{48}$ Another study assessed hopelessness reactivity to normal mood variations (eg, "When I feel down, I more often feel hopeless about everything") in a healthy sample of 381 participants and observed that it was positively associated with CAR AUCi; the authors controlled for sex, age, awakening time, work status, season, and cardiovascular disease, as well as sleep duration, smoking, and physical activity. ${ }^{41}$ Thus, while high endorsement of general hopelessness seems to be negatively associated with CAR, hopelessness reactivity to sad mood is positively associated. 


\section{Rumination and worry}

Thoughts and behaviors that repetitively focus one's attention on his or her negative feelings and consequences of those feelings ${ }^{49}$ as well as apprehensive expectations of possible negative outcomes in the future, ${ }^{35}$ maintain depressive mood and bring on a host of negative consequences. ${ }^{49}$ One study observed that high-trait self-focused rumination and less improvement of sad mood after induced distraction were both associated with a decreased CAR ${ }^{50}$ Furthermore, a sample of teachers endorsing high levels of work-related worry/ruminative thinking had flatter CAR the following morning compared to those with low levels of work-related rumination. ${ }^{51}$

\section{Subclinical depression}

Subclinical depression may represent a milder condition on the depression severity continuum ${ }^{52-54}$ as well as the precursor for the full disorder. ${ }^{55}$ Therefore, heightened subclinical depression is an important vulnerability factor for MDD.

Several studies specifically investigated association between subclinical levels of depression and CAR.

Dedovic et al separated young adult males and females into three groups - controls, and moderate and high subclinically depressed - and observed increased flattening of cortisol levels over the first hour with each increase in severity of subclinical depression. In addition, lower CAR AUCi was found in high subclinical depression compared to the control group, with moderate subclinical depression group showing AUCi levels in between these two groups. ${ }^{56}$

Another study by Mangold et al investigated 55 MexicanAmerican males and females (18-38) with low, moderate, and high subclinical depression symptoms. They showed that high compared to low-moderate subclinical depression symptoms were associated with flattening of the CAR. Further analyses revealed that attenuated delta between +30 minutes post-awakening vs awakening levels was a significant contributor to subclinical depressive symptomatology over and above emotional abuse, sexual abuse, general trauma, and physical punishment. ${ }^{57}$

On the other hand, a study examining young males, whose scores on the Hamilton Depression Inventory were within the low, nonclinical range, revealed a positive association between low, nonclinical depression levels and CAR AUCi. ${ }^{58}$ Similarly, high-trait negative affect has been associated with increased cortisol rise in the morning specifically in healthy men. ${ }^{18}$ Finally, null findings related to trait negativity in young women, ${ }^{59}$ and both trait negativity and depression scores in a large sample of community-dwelling men $^{60}$ have been reported.

\section{Family history of depression}

Another factor of high importance with respect to depression vulnerability is family history of depression. For example, recent meta-analysis revealed combined twofold (odds ratio $(\mathrm{OR})=2.14)$ increased familial risk of MDD for child and adult first-degree relatives of one proband with MDD. ${ }^{61}$ Earlier meta-analyses revealed slightly higher ORs: one focusing only on adults estimated an increased risk of $2.84,{ }^{62}$ while another, focusing specifically on child first-degree relatives of MDD probands, estimated the increased risk to be approximately threefold ( $\mathrm{OR}=3.21) \cdot{ }^{63}$ Not surprisingly, participants who had not been depressed themselves but have family history of depression represent an important model for depression vulnerability.

In a sample of 94 children, it was observed that maternal history of melancholic depression and child temperamental low positive emotionality were significantly associated with higher morning cortisol, assessed only at +30 minutes post-awakening. ${ }^{64}$ Two other studies examining healthy young adults observed similar effects. Specifically, those with family history of depression showed greater CAR levels in the first 30 minutes post-awakening compared to control participants. ${ }^{65}$ Furthermore, as compared with unaffected participants without parental history, unaffected individuals with diagnosed parental history of depression or anxiety showed a significantly higher CAR at +30 minutes, 45 minutes, and 60 minutes post-awakening, which was similar to that observed in the participants with depression or anxiety disorders. ${ }^{66}$ Therefore, having parental history of depression seems to be associated with higher CAR even if participants are currently asymptomatic.

\section{What process in the trajectory to depression does CAR index?}

Although associations have been found between CAR and various risk factors for depression, it still remains unclear which process in the trajectory to depression that CAR indexes? Along those lines, it has been proposed that CAR is an index of anticipation of stressors to come. For example, several studies have observed an increase in CAR on working day ${ }^{19,20}$ compared to weekend day. Some have suggested that anticipatory stress before going to work contributes to these increases in morning cortisol. For example, individuals who reported higher work overload scores and worry over the course of the last year showed more pronounced CAR on weekdays. ${ }^{20}$ Similarly, in a study of healthy undergraduate students, Zoccola et al observed that those participants who ruminated and/or worried the night before had higher CAR 
profile (both over time, AUCi, and delta change at 30 minutes post-awakening) compared to those who neither ruminated nor worried ${ }^{67}$ Along similar lines, increase in CAR may also be in response to negative mood the day before. For example, in a large sample of healthy older adults, prior-day feelings of loneliness, sadness, threat, and lack of control were associated with a higher cortisol level at +30 minutes post-awakening the next day. ${ }^{68}$ Furthermore, in a sample of adolescents oversampled for high levels of neuroticism, it was reported that higher levels of feeling lonely or sad the day before were associated with increased levels of CAR the following morning. ${ }^{69}$

It has also been suggested that CAR is rather an index of coping, although negative ${ }^{70}$ and null findings ${ }^{68}$ have been reported. Specifically, Powell and Schlotz showed that in a small healthy sample with a wide age range, higher CAR increases (assessed as CAR AUCi) were associated with attenuated distress responses to daily life stress assessed on multiple occasions throughout the day, on two consecutive weekdays, adjusting for age, sex, sleep quality, time of awakening, and oral contraceptive use; importantly, this effect was not due to prior-day CAR increases but specific for same-day CARs. In addition, no associations were found between stress anticipation and CARs. ${ }^{71}$ Other studies that assessed negative states only once a day and in the evening revealed that in adolescent females, increased CAR on a weekend was associated with higher levels of depression, anxiety, fatigue, and confusion in the evening of the same day, ${ }^{70}$ while in older participants, the CAR measure was not associated with states of loneliness, sadness, threat, and lack of control. ${ }^{68}$ Overall, despite some contradictory findings, CAR increase could be associated with successful coping with same-day daily life stress.

It is also important to note here that when it comes to the idea of CAR as an index of coping, one additional dimension of CAR should be taken into account - flexibility of the CAR. This dimension could potentially serve as an index of adaptation to demand or coping success over time but remains understudied. Indeed, only one study assessed CAR on 3 days (Sunday, Monday, and Tuesday) and examined associations between both CAR magnitude and CAR flexibility (weekend levels vs weekday levels) and levels of happiness, neuroticism, and perceived stress in healthy, employed males. ${ }^{72}$ When considering the CAR magnitude, as would be expected from the literature, inconsistent associations emerged; however, when CAR flexibility was assessed, a consistent pattern emerged. Specifically, resilient psychological profile (high happiness, low neuroticism, and low stress) was associated with a flexible CAR (lower CAR during weekends than during work days), while vulnerable profile (low happiness, high neuroticism, and high stress) was associated with a stiff CAR (same magnitude during weekdays and weekends). ${ }^{72}$

\section{CAR and depression}

As is the case between CAR measures and depression risk factors, depression itself has also been associated with both increased and lowered or blunted (nonsignificant, nominal change in) CAR. A large study investigating current middleaged depressed subjects found that, in comparison to control subjects, patients showed a higher CAR. ${ }^{73}$ Similar association was found in a sample of adolescent females ${ }^{74}$ as well as young adult women, currently suffering from depression. ${ }^{59}$ Higher CAR levels have also been observed in a remitted middle-aged depressed sample using medication, ${ }^{73}$ as well as in medication-free remitted depressed patients. ${ }^{75-77}$

However, others have observed a blunted CAR in young adult women with current mild-to-moderate clinical depression, ${ }^{78}$ as well as in older people with a depressive disorder. ${ }^{79}$ Further, in an outpatient population with major depression, more severe levels of depression were more likely associated with flattened diurnal cortisol patterns, ${ }^{80}$ and a lower CAR delta has been observed in depressed patients compared to patients with other psychiatric diagnoses. ${ }^{81}$

It has been suggested that the discrepancy between the findings might be related to depression severity, ${ }^{82}$ as well as how a depressive state is defined. For example, a recent study investigated association between depression levels, assessed as both a Diagnostic and Statistical Manual of Mental Disorders (DSM)-IV categorical variable and a dimension measure of a mood questionnaire, and CAR, in groups of outpatients (depressive, anxiety, and comorbid depressive and anxiety disorders) and controls. ${ }^{83}$ This approach revealed no group differences with respect to CAR when categorical distinctions between groups were applied. However, when nonlinear association was assessed across the whole sample, there was an inverted $U$ shape function explaining the association between anhedonic depression levels and AUC of the CAR, while controlling for the presence of different outpatient groups. Specifically, individuals with low or mild anhedonic depression levels showed similar CAR AUC as controls and those with moderate levels showed an increase, while those with severe levels showed a decrease in CAR AUC compared to controls. ${ }^{83}$ This association has since been replicated in a large sample of 1,029 participants with a lifetime depression and/or anxiety disorder from the Netherlands Study of Depression and Anxiety. ${ }^{84}$ The authors observed a 
significant nonlinear (inverted $U$ shape) association between CAR AUCi with general distress, anhedonic depression, and anxious arousal, even when controlling for confounders stemming from sociodemographic factors, cortisol sampling factors, and physical health of participants; the association was deemed generalizable to complete spectrum of healthy subjects, and current and remitted patients. ${ }^{84}$

\section{Prospective studies on CAR and depression}

Given the associations observed with both depressive risk factors and depressive episode or history of depression, effort has been exercised in investigating whether CAR levels may serve to prospectively predict depression onset. To this end, Adam et al investigated a large sample of 230 late adolescents to test whether CAR levels assessed across three consecutive typical weekdays can predict depression onset 1 year later. Indeed, they revealed that specifically higher CAR AUCi was associated with increased odds of having an episode over the follow-up period (with $\mathrm{OR}=3.0$ ); importantly, age and sex, health, baseline neuroticism, stressful life events, and past episodes of mood and anxiety disorders were included as covariates. In this study, the authors also assessed objective levels of stress experienced by the participants. The authors observed that introduction of CAR did not reduce the association between life stress levels in the past year and the development of MDD, which suggested that CAR is independently associated with the development of MDD and is likely not a mediator of the association between recent life stress events and the development of MDD. ${ }^{85}$

By continuing the follow-up of this cohort, VrshekSchallhorn et al showed that higher levels of CAR at baseline predicted major depressive episodes (MDEs) for 2.5 years following cortisol measurement. ${ }^{86}$ Notably, elevation in CAR did not significantly increase vulnerability to prospective objective major stressful life events but did predict depressive episode recurrences more strongly than first onsets. Specifically, CAR magnitude was similar between individuals suffering from first-onset MDE or recurrent MDE; however, CAR appeared particularly important in predicting whether someone with history of depression will experience a new MDE. The authors suggested that a high CAR may represent a time-limited risk factor for onsets of MDEs, which increases risk for depression being independent of future major stressful life events. ${ }^{86}$ This time-limited association between CAR and depression is inadvertently supported by another study examining a sample of 841 healthy 15 -year olds that found no association between CAR levels at baseline and development of depression 3 years later (at 18 years of age). ${ }^{87}$

Finally, Hardeveld et al assessed whether HPA axis parameters including CAR, DST, and evening cortisol, obtained at baseline, predict time to recurrence in 549 subjects with a lifetime diagnosis of MDD in remission for at least 6 months preceding the baseline assessment. Specifically, at baseline, on day 1, participants completed salivary samples at home to establish the CAR profile and the diurnal rhythm. Then, after the last sample (collected at $11 \mathrm{pm}$ ) on day 1 , participants ingested $0.5 \mathrm{mg}$ of DEX. The DEX suppression was measured using the cortisol sampling the next morning upon awakening. Time to the first new MDD episode was assessed prospectively at 2-year and 4-year follow-up. In this study sample, $41.3 \%$ had no depressive symptoms in the 5 years preceding baseline, and only $51.4 \%$ had one MDE in the past, which suggests that participants in this study did not have a chronic recurrent course in the recent past. A Cox regression analysis revealed that higher CAR AUCi was associated with time to recurrence of MDD and that this relationship was not dependent on objective number of stressful life events that occurred prior to the baseline assessment; evening cortisol levels and cortisol suppression after DEX ingestion were not related to time to recurrence. ${ }^{88}$ Here, it is noteworthy that the lack of association between recurrence and cortisol levels in response to DST test might be due to the lack of sensitivity of the test. ${ }^{89,90}$ Indeed, the current gold standard is the dexamethasone/corticotrophin-releasing hormone (DEX/CRH) test, ${ }^{89,90}$ and it has been shown that persisting cortisol hypersecretion on DEX/CRH test enhances the risk for relapse within the following 6 months. ${ }^{91,92}$ Overall, this study suggests that among those who have previously experienced a depressive episode, those with higher CAR are more vulnerable to recurrence of MDD.

\section{Conclusion}

The work to date reveals that CAR is highly heritable which indicates that to a certain degree, it is a trait-like feature; however, as it is also greatly influenced by environmental and situational factors, it can also serve as an index of one's overall vulnerability to depression as it relates to stressful experiences, particularly with respect to daily stressors. Results from the longitudinal studies showing increased CAR being linked with depression onset and recurrence, independently of stressful life events, seem to be at odds with this hypothesis. However, it is worth noting that these studies assessed number of occurrences of objective stressful experiences, in the last year. Yet, when it comes to the 
link between stress and depression, it is not only the number of major stressful events experienced but also the level of daily hassles experienced,,$^{93,94}$ as well as type of stress (eg, personally targeted social stressors are more salient) ${ }^{95}$ and one's subjective or perceived levels of stress and coping abilities that are of importance. Thus, CAR might not mediate or moderate the association between number or severity of objective major stressors and depression onset, but rather, it might be more attune to experiences of daily hassles as well as to the degree to which one feels stressed or threatened, and particularly, to the degree that one can cope with a stressor (major or minor). In that case, CAR would also be particularly relevant when it comes to recurrence of depression (as has been shown ${ }^{86}$ ), given that the onset of the first depressive episode is often linked to experience of a major stressful event, while the subsequent episodes could be triggered by more minor events, due to stress sensitization. ${ }^{96}$

The results to date suggest that in the healthy populations, increased CAR is particularly relevant to being able to meet specific demands of the upcoming day (eg, workday vs weekend), and decrease of stress on that specific day, and therefore has been linked to coping. One could speculate that this link may become dysregulated once coping employed is inefficient in eliminating feeling of stress over time. In that case, heightened CAR (and possibly inflexible or stiff CAR) may switch from signaling coping to signaling anticipation of stress of the next day. As previously suggested, ${ }^{84}$ it is likely that at a certain threshold, persistent increased CAR may be downregulated, and become blunted. This could account for why some studies have observed heighted CAR and others blunted CAR to be related to poor mental health profile as related to depression.

Both of these hypotheses need to be tested in future studies. Overall, the studies reveal a complex and important association between CAR and vulnerability to depression.

\section{Disclosure}

The authors have no conflicts of interest to disclose.

\section{References}

1. Binder EB, Nemeroff CB. The CRF system, stress, depression and anxiety-insights from human genetic studies. Mol Psychiatry. 2010; 15(6):574-588.

2. Burke HM, Davis MC, Otte C, Mohr DC. Depression and cortisol responses to psychological stress: a meta-analysis. Psychoneuroendocrinology. 2005;30(9):846-856.

3. Holsboer F. The corticosteroid receptor hypothesis of depression. Neuropsychopharmacology. 2000;23(5):477-501.

4. Hatzinger M, Brand S, Perren S, et al. Pre-schoolers suffering from psychiatric disorders show increased cortisol secretion and poor sleep compared to healthy controls. J Psychiatr Res. 2012;46(5):590-599.
5. Holsboer F, Ising M. Stress hormone regulation: biological role and translation into therapy. Annu Rev Psychol. 2010;61(81-109): C101-C111.

6. Cowen PJ. Not fade away: the HPA axis and depression. Psychol Med. 2010;40(1):1-4.

7. Kudielka BM, Hawkley LC, Adam EK, Cacioppo JT. Compliance with ambulatory saliva sampling in the chicago health, aging, and social relations study and associations with social support. Ann Behav Med. 2007;34(2):209-216.

8. Wilhelm I, Born J, Kudielka BM, Schlotz W, Wust S. Is the cortisol awakening rise a response to awakening? Psychoneuroendocrinology. 2007;32(4):358-366.

9. Fries E, Dettenborn L, Kirschbaum C. The cortisol awakening response (CAR): facts and future directions. Int J Psychophysiol. 2009; 72(1):67-73.

10. Clow A, Hucklebridge F, Stalder T, Evans P, Thorn L. The cortisol awakening response: more than a measure of HPA axis function. Neurosci Biobehav Rev. 2009;35(1):97-103.

11. Pruessner JC, Wolf OT, Hellhammer DH, et al. Free cortisol levels after awakening: a reliable biological marker for the assessment of adrenocortical activity. Life Sci. 1997;61(26):2539-2549.

12. Wust S, Federenko I, Hellhammer DH, Kirschbaum C. Genetic factors, perceived chronic stress, and the free cortisol response to awakening. Psychoneuroendocrinology. 2000;25(7):707-720.

13. Hucklebridge F, Hussain T, Evans P, Clow A. The diurnal patterns of the adrenal steroids cortisol and dehydroepiandrosterone (DHEA) in relation to awakening. Psychoneuroendocrinology. 2005;30(1): $51-57$.

14. Wust S, Wolf J, Hellhammer DH, Federenko I, Schommer N, Kirschbaum C. The cortisol awakening response - normal values and confounds. Noise Health. 2000;2(7):79-88.

15. Buijs RM, Wortel J, Van Heerikhuize JJ, Kalsbeek A. Novel environment induced inhibition of corticosterone secretion: physiological evidence for a suprachiasmatic nucleus mediated neuronal hypothalamoadrenal cortex pathway. Brain Res. 1997;758(1-2):229-236.

16. Edwards S, Evans P, Hucklebridge F, Clow A. Association between time of awakening and diurnal cortisol secretory activity. Psychoneuroendocrinology. 2001;26(6):613-622.

17. Kudielka BM, Kirschbaum C. Awakening cortisol responses are influenced by health status and awakening time but not by menstrual cycle phase. Psychoneuroendocrinology. 2003;28(1):35-47.

18. Polk DE, Cohen S, Doyle WJ, Skoner DP, Kirschbaum C. State and trait affect as predictors of salivary cortisol in healthy adults. Psychoneuroendocrinology. 2005;30(3):261-272.

19. Kunz-Ebrecht SR, Kirschbaum C, Marmot M, Steptoe A. Differences in cortisol awakening response on work days and weekends in women and men from the Whitehall II cohort. Psychoneuroendocrinology. 2004;29(4):516-528.

20. Schlotz W, Hellhammer J, Schulz P, Stone AA. Perceived work overload and chronic worrying predict weekend-weekday differences in the cortisol awakening response. Psychosom Med. 2004;66(2):207-214.

21. Stalder T, Hucklebridge F, Evans P, Clow A. Use of a single case study design to examine state variation in the cortisol awakening response: relationship with time of awakening. Psychoneuroendocrinology. 2009; 34(4):607-614.

22. Matchock RL, Dorn LD, Susman EJ. Diurnal and seasonal cortisol, testosterone, and DHEA rhythms in boys and girls during puberty. Chronobiol Int. 2007;24(5):969-990.

23. Badrick E, Kirschbaum C, Kumari M. The relationship between smoking status and cortisol secretion. J Clin Endocrinol Metab. 2007; 92(3):819-824.

24. Federenko I, Wust S, Hellhammer DH, Dechoux R, Kumsta R, Kirschbaum C. Free cortisol awakening responses are influenced by awakening time. Psychoneuroendocrinology. 2004;29(2):174-184.

25. Bartels M, de Geus EJ, Kirschbaum C, Sluyter F, Boomsma DI. Heritability of daytime cortisol levels in children. Behav Genet. 2003;33(4):421-433. 
26. Gustafsson PA, Gustafsson PE, Anckarsäter H, et al. Heritability of cortisol regulation in children. Twin Res Hum Genet. 2011; 14(6):553-561.

27. Kupper N, de Geus EJ, van den Berg M, Kirschbaum C, Boomsma DI, Willemsen G. Familial influences on basal salivary cortisol in an adult population. Psychoneuroendocrinology. 2005;30(9):857-868.

28. Franz CE, York TP, Eaves LJ, et al. Genetic and environmental influences on cortisol regulation across days and contexts in middle-aged men. Behav Genet. 2010;40(4):467-479.

29. Clow A, Law R, Evans P, et al. Day differences in the cortisol awakening response predict day differences in synaptic plasticity in the brain. Stress. 2014;17(3):219-223.

30. Menet JS, Rosbash M. When brain clocks lose track of time: cause or consequence of neuropsychiatric disorders. Curr Opin Neurobiol. 2011;21(6):849-857.

31. Nader N, Chrousos GP, Kino T. Interactions of the circadian CLOCK system and the HPA axis. Trends Endocrinol Metab. 2010; 21(5):277-286

32. Grav S, Stordal E, Romild UK, Hellzen O. The relationship among neuroticism, extraversion, and depression in the HUNT Study: in relation to age and gender. Issues Ment Health Nurs. 2012;33(11): 777-785.

33. Cuijpers P, Smit F, Willemse G. Predicting the onset of major depression in subjects with subthreshold depression in primary care: a prospective study. Acta Psychiatr Scand. 2005;111(2):133-138.

34. De Graaf R, Bijl RV, Ravelli A, Smit F, Vollebergh WA. Predictors of first incidence of DSM-III-R psychiatric disorders in the general population: findings from the Netherlands Mental Health Survey and Incidence Study. Acta Psychiatr Scand. 2002;106(4):303-313.

35. Roelofs J, Huibers M, Peeters F, Arntz A, van Os J. Rumination and worrying as possible mediators in the relation between neuroticism and symptoms of depression and anxiety in clinically depressed individuals. Behav Res Ther. 2008;46(12):1283-1289.

36. Portella MJ, Harmer CJ, Flint J, Cowen P, Goodwin GM. Enhanced early morning salivary cortisol in neuroticism. Am J Psychiatry. 2005; 162(4):807-809.

37. Madsen KS, Jernigan TL, Iversen $P$, et al. Cortisol awakening response and negative emotionality linked to asymmetry in major limbic fibre bundle architecture. Psychiatry Res. 2012;201(1):63-72.

38. Mangold D, Mintz J, Javors M, Marino E. Neuroticism, acculturation and the cortisol awakening response in Mexican American adults. Horm Behav. 2012;61(1):23-30.

39. Hauner KK, Adam EK, Mineka S, et al. Neuroticism and introversion are associated with salivary cortisol patterns in adolescents. Psychoneuroendocrinology. 2008;33(10):1344-1356.

40. Laceulle OM, Nederhof E, van Aken MAG, Ormel J. Adolescent personality: associations with basal, awakening, and stress-induced cortisol responses. J Pers. In press 2014.

41. van Santen A, Vreeburg SA, Van der Does AJ, Spinhoven P, Zitman FG, Penninx BW. Psychological traits and the cortisol awakening response: results from the Netherlands Study of Depression and Anxiety. Psychoneuroendocrinology. 2011;36(2):240-248.

42. Hill EM, Billington R, Krageloh C. The cortisol awakening response and the big five personality dimensions. Pers Individ Dif. 2013; 55:600-605.

43. Chan SW, Goodwin GM, Harmer CJ. Highly neurotic never-depressed students have negative biases in information processing. Psychol Med. 2007;37(9):1281-1291.

44. Garcia-Banda G, Chellew K, Fornes J, Perez G, Servera M, Evans P. Neuroticism and cortisol: pinning down an expected effect. Int J Psychophysiol. 2014;91(2):132-138.

45.Abramson LY, Metalsky GI, Alloy LB. Hopelessness depression: a theory-based subtype of depression. Psychol Rev. 1989;96(2):358-372.

46. Kwon P, Laurenceau JP. A longitudinal study of the hopelessness theory of depression: testing the diathesis-stress model within a differential reactivity and exposure framework. J Clin Psychol. 2002;58(10):1305-1321.
47. Barnhofer T, Chittka T. Cognitive reactivity mediates the relationship between neuroticism and depression. Behav Res Ther. 2010; 48(4):275-281.

48. Sjogren E, Leanderson P, Kristenson M. Diurnal saliva cortisol levels and relations to psychosocial factors in a population sample of middle-aged Swedish men and women. Int J Behav Med. 2006;13(3):193-200.

49. Lyubomirsky $\mathrm{S}, \mathrm{Tkach} \mathrm{C}$. The consequences of dysphoric rumination. In: Papageorgiou C, Wells A, editors. Depressive Rumination: Nature, Theory and Treatment. West Sussez, England: John Wiley \& Sons Ltd; 2004:298.

50. Kuehner C, Holzhauer S, Huffziger S. Decreased cortisol response to awakening is associated with cognitive vulnerability to depression in a nonclinical sample of young adults. Psychoneuroendocrinology. 2007;32(2):199-209.

51. Cropley M, Rydstedt LW, Devereux JJ, Middleton B. The relationship between work-related rumination and evening and morning salivary cortisol secretion. Stress Health. In press 2013.

52. Solomon A, Haaga DA, Arnow BA. Is clinical depression distinct from subthreshold depressive symptoms? A review of the continuity issue in depression research. J Nerv Ment Dis. 2001;189(8):498-506.

53. Lewinsohn PM, Klein DN, Durbin EC, Seeley JR, Rohde P. Family study of subthreshold depressive symptoms: risk factor for MDD? J Affect Disord. 2003;77(2):149-157.

54. Rivas-Vazquez RA, Saffa-Biller D, Ruiz I, Blais MA, Rivas-Vazquez A. Current issues in anxiety and depression: comorbid, mixed, and subthreshold disorders. Prof Psychol Res Pract. 2004;35(1):74-83.

55. Shankman SA, Lewinsohn PM, Klein DN, Small JW, Seeley JR, Altman SE. Subthreshold conditions as precursors for full syndrome disorders: a 15-year longitudinal study of multiple diagnostic classes. J Child Psychol Psychiatry. 2009;50(12):1485-1494.

56. Dedovic K, Engert V, Duchesne A, et al. Cortisol awakening response and hippocampal volume: vulnerability for major depressive disorder? Biol Psychiatry. 2010;68(9):847-853

57. Mangold D, Marino E, Javors M. The cortisol awakening response predicts subclinical depressive symptomatology in Mexican American adults. J Psychiatr Res. 2011;45(7):902-909.

58. Pruessner M, Hellhammer DH, Pruessner JC, Lupien SJ. Self-reported depressive symptoms and stress levels in healthy young men: associations with the cortisol response to awakening. Psychosom Med. 2003;65(1):92-99.

59. Dienes KA, Hazel NA, Hammen CL. Cortisol secretion in depressed, and at-risk adults. Psychoneuroendocrinology. 2013;38(6):927-940.

60. Doane LD, Franz CE, Prom-Wormley E, et al. Negative emotionality, depressive symptoms and cortisol diurnal rhythms: analysis of a community sample of middle-aged males. Horm Behav. 2011;60(2): 202-209.

61. Wilde A, Chan HN, Rahman B, et al. A meta-analysis of the risk of major affective disorder in relatives of individuals affected by major depressive disorder or bipolar disorder. J Affect Disord. 2014;158:37-47.

62. Sullivan PF, Neale MC, Kendler KS. Genetic epidemiology of major depression: review and meta-analysis. Am J Psychiatry. 2000; 157(10):1552-1562.

63. Rice F, Harold G, Thapar A. The genetic aetiology of childhood depression: a review. J Child Psychol Psychiatry. 2002;43(1):65-79.

64. Dougherty LR, Klein DN, Olino TM, Dyson M, Rose S. Increased waking salivary cortisol and depression risk in preschoolers: the role of maternal history of melancholic depression and early child temperament. J Child Psychol Psychiatry. 2009;50(12):1495-1503.

65. Mannie ZN, Harmer CJ, Cowen PJ. Increased waking salivary cortisol levels in young people at familial risk of depression. Am J Psychiatry. 2007;164(4):617-621.

66. Vreeburg SA, Hartman CA, Hoogendijk WJ, et al. Parental history of depression or anxiety and the cortisol awakening response. $\mathrm{Br} J$ Psychiatry. 2010;197(3):180-185.

67. Zoccola PM, Dickerson SS, Yim IS. Trait and state perseverative cognition and the cortisol awakening response. Psychoneuroendocrinology. 2011;36(4):592-595. 
68. Adam EK, Hawkley LC, Kudielka BM, Cacioppo JT. Day-to-day dynamics of experience - cortisolassociations in a population-based sampleof older adults. PNAS. 2006;103(45):17058-17063.

69. Doane LD, Adam EK. Loneliness and cortisol: momentary, dayto-day, and trait associations. Psychoneuroendocrinology. 2010; 35(3):430-441.

70. Shibuya I, Nagamitsu S, Okamura H, et al. High correlation between salivary cortisol awakening response and the psychometric profiles of healthy children. Biopsychosoc Med. 2014;8(1):1-4.

71. Powell DJ, Schlotz W. Daily life stress and the cortisol awakening response: testing the anticipation hypothesis. PLoS One. 2012;7(12):e52067.

72. Mikolajczak M, Quoidbach J, Vanootighem V, et al. Cortisol awakening response (CAR)'s flexibility leads to larger and more consistent associations with psychological factors than CAR magnitude. Psychoneuroendocrinology. 2010;35(5):752-757.

73. Vreeburg SA, Hoogendijk WJ, van Pelt J, et al. Major depressive disorder and hypothalamic-pituitary-adrenal axis activity: results from a large cohort study. Arch Gen Psychiatry. 2009;66(6):617-626.

74. Ulrike S, Reinhold L, Dirk H. Major depression in young girls is related to altered cortisol awakening response. Eur Child Adolesc Psychiatry. 2013;22(6):379-384

75. Bhagwagar Z, Hafizi S, Cowen PJ. Increase in concentration of waking salivary cortisol in recovered patients with depression. Am J Psychiatry. 2003;160(10):1890-1891.

76. Bhagwagar Z, Hafizi S, Cowen PJ. Increased salivary cortisol after waking in depression. Psychopharmacology. 2005;182(1):54-57.

77. Aubry JM, Jermann F, Gex-Fabry M, et al. The cortisol awakening response in patients remitted from depression. J Psychiatr Res. 2010;44(16):1199-1204.

78. Stetler C, Miller GE. Blunted cortisol response to awakening in mild to moderate depression: regulatory influences of sleep patterns and social contacts. J Abnorm Psychol. 2005;114(4):697-705.

79. Rhebergen D, Korten NC, Penninx BW, et al. Hypothalamic-pituitaryadrenal axis activity in older persons with and without a depressive disorder. Psychoneuroendocrinology. 2015;51:341-350.

80. Hsiao FH, Yang TT, Ho RT, et al. The self-perceived symptom distress and health-related conditions associated with morning to evening diurnal cortisol patterns in outpatients with major depressive disorder. Psychoneuroendocrinology. 2009;35(4):503-515.

81. Huber TJ, Issa K, Schik G, Wolf OT. The cortisol awakening response is blunted in psychotherapy inpatients suffering from depression. Psychoneuroendocrinology. 2006;31(7):900-904.

82. Chida Y, Steptoe A. Cortisol awakening response and psychosocial factors: a systematic review and meta-analysis. Biol Psychol. 2009;80(3):265-278.
83. Veen G, van Vliet IM, Derijk RH, Giltay EJ, van Pelt J, Zitman FG. Basal cortisol levels in relation to dimensions and DSM-IV categories of depression and anxiety. Psychiatry Res. 2010;185(1-2):121-128.

84. Wardenaar KJ, Vreeburg SA, van Veen T, et al. Dimensions of depression and anxiety and the hypothalamo-pituitary-adrenal axis. Biol Psychiatry. 2011;69(4):366-373.

85. Adam EK, Doane LD, Zinbarg RE, Mineka S, Craske MG, Griffith JW. Prospective prediction of major depressive disorder from cortisol awakening responses in adolescence. Psychoneuroendocrinology. 2010;35(6):921-931.

86. Vrshek-Schallhorn S, Doane LD, Mineka S, Zinbarg RE, Craske MG, Adam EK. The cortisol awakening response predicts major depression: predictive stability over a 4-year follow-up and effect of depression history. Psychol Med. 2013;43(3):483-493.

87. Carnegie R, Araya R, Ben-Shlomo Y, et al. Cortisol awakening response and subsequent depression: prospective longitudinal study. Br J Psychiatry. 2014;204(2):137-143.

88. Hardeveld F, Spijker J, Vreeburg SA, et al. Increased cortisol awakening response was associated with time to recurrence of major depressive disorder. Psychoneuroendocrinology. 2014;50:62-71.

89. Ising M, Kunzel HE, Binder EB, Nickel T, Modell S, Holsboer F. The combined dexamethasone/CRH test as a potential surrogate marker in depression. Prog Neuropsychopharmacol Biol Psychiatry. 2005;29(6):1085-1093.

90. Watson S, Gallagher P, Smith MS, Ferrier IN, Young AH. The dex/ $\mathrm{CRH}$ test - is it better than the DST? Psychoneuroendocrinology. 2006;31(7):889-894.

91. Zobel AW, Nickel T, Sonntag A, Uhr M, Holsboer F, Ising M. Cortisol response in the combined dexamethasone/CRH test as predictor of relapse in patients with remitted depression. A prospective study. J Psychiatr Res. 2001;35(2):83-94.

92. Zobel AW, Yassouridis A, Frieboes RM, Holsboer F. Prediction of medium-term outcome by cortisol response to the combined dexamethasone-CRH test in patients with remitted depression. $\mathrm{Am}$ J Psychiatry. 1999;156(6):949-951.

93. Hutchinson JG, Williams PG. Neuroticism, daily hassles, and depressive symptoms: an examination of moderating and mediating effects. Pers Individ Dif. 2007;42:1367-1378.

94. Sher L. Daily hassles, cortisol, and the pathogenesis of depression. Med Hypotheses. 2004;62(2):198-202.

95. Slavich GM, Thornton T, Torres LD, Monroe SM, Gotlib IH. Targeted rejection predicts hastened onset of major depression. J Soc Clin Psychol. 2009;28(2):223-243.

96. Stroud CB, Davila J, Hammen C, Vrshek-Schallhorn S. Severe and nonsevere events in first onsets versus recurrences of depression: evidence for stress sensitization. J Abnorm Psychol. 2011;120(1):142-154.
Neuropsychiatric Disease and Treatment

\section{Publish your work in this journal}

Neuropsychiatric Disease and Treatment is an international, peerreviewed journal of clinical therapeutics and pharmacology focusing on concise rapid reporting of clinical or pre-clinical studies on a range of neuropsychiatric and neurological disorders. This journa is indexed on PubMed Central, the 'PsycINFO' database and CAS,

\section{Dovepress}

and is the official journal of The International Neuropsychiatric Association (INA). The manuscript management system is completely online and includes a very quick and fair peer-review system, which is all easy to use. Visit http://www.dovepress.com/testimonials.php to read real quotes from published authors. 